\title{
INVERSIÓN EXTRANJERA DIRECTA Y CADENAS AGROINDUSTRIALES DE COSTA RICA: HACIA UNA TIPOLOGÍA ${ }^{1}$
}

\section{FOREIGN DIRECT INVESTMENT AND AGRIBUSINESS CHAINS IN COSTA RICA: TOWARD A TYPOLOGY}

\author{
Ivania García Cascante ${ }^{2}$ \\ Jorge Andrey Valenciano Salazar ${ }^{3}$
}

\begin{abstract}
Resumen
Mediante el cálculo de un índice global de desarrollo humano y con base en datos de los censos 2000 y 2011, este artículo evidencia como en los distritos con una presencia más fuerte de inversión extranjera directa (IED), es decir, distritos productores de piña y banano se concentran en posiciones de desarrollo social y económico menor que aquellos donde existe inversión nacional o mixta, los cuales se concentran en posiciones intermedias, es decir, distritos productores de café y flores. Esta condición se debe a la gobernanza que ejercen las empresas más grandes sobre las agrocadenas, lo que genera una situación de dependencia de los pequeños y medianos productores para poder comercializar sus productos, así como un uso intensivo de factores de producción poco remunerados, como la mano de obra.
\end{abstract}

${ }^{1}$ Doi: http://dx.doi.org/10.15359/eys.21-49.3

Recibido el 5 de mayo de 2016. Reenvíos: 6 de junio de 2016, 17 de junio de 2016. Aceptado el 20 de junio de 2016. Publicado el 24 de junio de 2016.

${ }^{1}$ El siguiente artículo se escribió dentro de los siguientes proyectos desarrollados en la Escuela de Economía y Centro Internacional de Política Económica de la Universidad Nacional: a) Estructuras de control en las cadenas y flujos de financiamiento (IED y crédito): Posibilidades para los productores nacionales, código 022004. B) Efecto de las organizaciones de economía solidaria y comercio justo sobre el desarrollo económico y social de las comunidades en Costa Rica, código SIA 0110-15.

${ }^{2}$ Economista. Investigadora, Escuela de Economía, Universidad Nacional, Costa Rica. Correo electrónico: ivaniagc@hotmail.com

${ }^{3}$ Economista. Académico e Investigador, Escuela de Economía, Universidad Nacional, Costa Rica. Correo electrónico:: iorge.valenciano.salazar@una.ac.cr

Ivania García Cascante, Jorge Andrey Valenciano Salazar

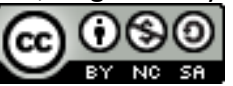

Revista Economía y Sociedad by Universidad Nacional is licensed under a Creative Commons Reconocimiento-NoComercial- 
Palabras claves: agrocadenas; gobernanza; inversión extrajera directa; desarrollo

\begin{abstract}
Using an overall human development index and data from the 2000 and 2011 census, this paper shows how districts with a stronger FDI presence (that is districts producing pineapple and bananas) are concentrated in social and economic development positions lower than those districts with mixed or national investment, which are concentrated in intermediate positions (that is districts producing coffee and flowers). This condition is due to the governance exercised by larger companies on the agricultural chains, which generates dependence from small and medium producers to market their products as well as intensive use of low-paid production factors such as workforce.
\end{abstract}

Keywords: agricultural chains; governance; foreign direct investment; development

\title{
1. Introducción
}

En el presente artículo se analizan las agrocadenas de cuatro productos importantes para la economía costarricense: piña, banano, flores y café. El común denominador de estas mercancías es que sus cadenas son dinamizadas por la inversión extranjera directa (IED). Además, ubican geográficamente su eslabón de producción primario en las regiones rurales.

El objetivo de este capítulo es mostrar la evolución del estado de desarrollo de dichos espacios rurales para aproximar tipologías de desarrollo en distritos dinamizados por la IED. Para ello se utilizan indicadores sociales y económicos de los Censos Nacionales de los años 2000 y 2011, conjugados con una aproximación de la estructura productiva de dichos medios rurales en los cuales se desarrollan pequeños productores, cooperativas y empresas transnacionales, entre otros agentes de las cadenas en las regiones correspondientes.

\section{Conceptos y metodología}

\subsection{Los efectos esperados de la inversión extranjera directa}

Uno de los principales efectos de peso que han sido señalados en la implementación de políticas de atracción de IED es el impacto en el crecimiento de la economía. Con respecto a ello, Cubero (2006) señala tres posibles formas para generar dicho crecimiento:

2

Ivania García Cascante, Jorge Andrey Valenciano Salazar

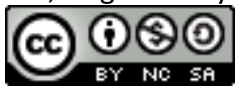

Revista Economía y Sociedad by Universidad Nacional is licensed under a Creative Commons Reconocimiento-NoComercial- 
- La inversión física: mediante la inversión fija de las compañías extranjeras y el gasto de capital de las empresas domésticas.

- El trabajo: mediante la generación de empleos, el entrenamiento a los empleados y los salarios.

- La productividad de los factores: mediante los efectos directos de las empresas extranjeras.

Sin embargo, aunque se señalen las bondades de la IED en el crecimiento económico, estas no deben confundirse con el desarrollo. Se dice que hay crecimiento económico cuando las variables como la producción y el consumo aumentan, pero esto no significa necesariamente que hay una mejora en el bienestar o en la calidad de vida de las personas que viven en las regiones rurales.

De hecho, estudios como el de Gallagher y López (2008) han señalado que la IED no es suficiente para generar crecimiento de manera sustentable, dado que la misma no es un fin, sino un medio para ello. Sobre todo la IED que se lleva a cabo en los países en busca de recursos como fertilidad de la tierra y mano de obra, muchas veces realizan sus procesos productivos sin tomar en cuenta las externalidades ambientales negativas que generan, es por ello que estos autores han señalado también que las políticas encaminadas hacia la atracción de IED deben acompañarse de políticas dirigidas a incrementar la capacidad de las empresas nacionales y de políticas para establecer estándares de protección ambiental.

Por su parte, Díaz y Valenciano (2011) consideran que a pesar de las múltiples ventajas de la IED, tales como: inversión física, formación del capital humano, externalidades, efectos sobre las exportaciones e importaciones, entre otras; es necesario que la atracción de la IED incorpore estrategias para crear encadenamientos con las empresas locales. Ese resultado, sin embargo, es complejo, se requiere de políticas nacionales y estrategias orientadas por las transnacionales para generar un grupo de encadenamientos.

\subsection{Indicadores de análisis planteados}

Para lograr los objetivos propuestos a nivel empírico se hace uso de los siguientes recursos:

1. A partir de la teoría de cadenas globales de valor, se caracteriza la estructura general de cada una de las cadenas de los cuatro productos bajo estudio. Se estudia el posicionamiento internacional de los agentes presentes en cada cadena y los vínculos con los medios rurales a través de la revisión bibliográfica de trabajos previos.

Ivania García Cascante, Jorge Andrey Valenciano Salazar

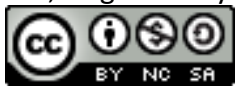

Revista Economía y Sociedad by Universidad Nacional is licensed under a Creative Commons Reconocimiento-NoComercial- 
2. Se aproxima un modelo de tipologías de desarrollo a partir de indicadores sociales y económicos aportados por los censos 2000 y 2011. Se considera información acerca de vivienda, empleo, educación, disponibilidad de artículos en el hogar y servicios básicos para instrumentalizar una medida del estado actual del desarrollo de los distritos que producen los bienes de interés, en comparación con el resto de distritos distintos del país. A partir de estos datos se construyen índices cuyo uso se fundamenta en la metodología del Biograma del IICA (Sepúlveda, S; 2008), tal como se explica a continuación:

Los indicadores sociales contemplan servicios básicos (porcentaje de viviendas con acueducto y electricidad), viviendas en buen estado y hacinadas, asistencia a la educación secundaria (el porcentaje de población de 15 años o más con al menos un año de educación secundaria) y acceso a seguridad social (porcentaje de población no asegurada); mientras que, por parte de los indicadores económicos incluyen la tasa de ocupación, tasa de desempleo y la tenencia de artículos (porcentaje de viviendas que poseen computadora y teléfono fijo). Se debe aclarar que estos indicadores permiten comparar los distritos en cada año, es decir, metodológicamente no se deben hacer comparaciones intertemporales, por lo que se procederá a analizar la calificación de cada distrito en cada uno de los años analizados ${ }^{4}$.

A partir de los indicadores explicados anteriormente se calcula el Índice Global de Desarrollo Humano (IGDH), que se compone de los subíndices económico y social. En primera instancia se calcularon los índices sociales, económicos y el global de 460 distritos de Costa Rica, para el 2000 y en el 2011. En cada año se estableció la posición de cada distrito, lo cual permitió elaborar una jerarquización (ranking) de distritos, en donde se evidenciaron ascensos o caídas. No se incluyeron variables ambientales en el índice, ya que los censos proporcionados por el

\footnotetext{
${ }^{4}$ Los indicadores fueron seleccionados según dos elementos. Elemento 1: disponibilidad de información homogénea a nivel distrital para todas las localidades estudiadas durante dos periodos temporales. Al momento de la investigación, solo el Censo de Población del INEC de los años 2000 y 2011 cumplían con dichos requisitos. Elemento 2: se tomaron los indicadores homogéneos que fueran distritales tanto a nivel social como económico. Los indicadores sociales son de diferente naturaleza, de tal manera que se pueda calcular un índice global del desempeño social, de esa forma tenemos: 1. En acceso a servicios se escogió: 1.1 Servicios básicos: porcentaje de viviendas con acueducto y electricidad, 1.2 Acceso a seguridad social (porcentaje de población no asegurada) 2. En infraestructura básica a nivel familiar, se escogió: 2.1 Tenencia de viviendas en buen estado y hacinadas. 3. La educación como una variable de accenso social se escogió: 3.1 Asistencia a la educación secundaria (el porcentaje de población de 15 años o más con al menos un año de educación secundaria.

En cuanto a los indicadores económicos también se escogieron de tal manera se tenga una idea general del desempeño distrital, estos indicadores son: 1. Empleo, se tienen los siguientes indicadores 1.1 La tasa de ocupación, 1.2 Tasa de desempleo; la variable 2 es el desempeño socioeconómico e infraestructura para lo cual se plantearon los siguientes indicadores: 2.1 La tenencia de artículos (porcentaje de viviendas que poseen computadora y teléfono fijo.

La tenencia de computadora no distingue si es portátil o de escritorio, ya que las estadísticas del INEC hacen una diferenciación solamente para el censo del año 2012.
}

4

Ivania García Cascante, Jorge Andrey Valenciano Salazar

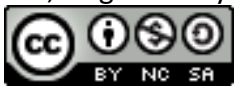

Revista Economía y Sociedad by Universidad Nacional is licensed under a Creative Commons Reconocimiento-NoComercial- 
Instituto Nacional de Estadísticas y Censos no manejan este tipo de variables en términos geográficos.

Para aproximar el estado del desarrollo de los espacios rurales se empleó la metodología conocida como "Biograma" (Sepúlveda, 2008), cuya fórmula se basa en estandarización de indicadores, que posteriormente serán incorporados en la construcción de un índice de desarrollo. Asimismo, como fortalezas de esta metodología se tiene que se puede analizar una misma unidad en diferentes periodos.

En primer lugar, se debe establecer si el indicador tiene una relación positiva o negativa con el desarrollo y según esto, se elegirá la forma de estandarización:

Donde,

$$
\begin{array}{ll}
\text { Relación positiva (+) } & (\mathrm{x}-\mathrm{m}) /(\mathrm{M}-\mathrm{m}) \\
\text { Relación negativa (-) } & (\mathrm{x}-\mathrm{M}) /(\mathrm{m}-\mathrm{M})
\end{array}
$$

$\boldsymbol{x}$ es el indicador en un periodo de tiempo $t$

$\boldsymbol{m}$ es el valor mínimo existente en todas las unidades de análisis de periodo t

M es el valor máximo existente en todas las unidades de análisis de periodo $t$

Los valores que alcanza el índice varían entre 0 y 1 , donde 0 será la peor situación o estado del desarrollo y 1 será el mejor. Sin embargo, el Biograma establece varios estados del desarrollo, los cuales se representan a continuación:

Tabla 1.

\begin{tabular}{|c|c|c|c|c|c|}
\hline \multicolumn{5}{|c|}{ Escala } & \multirow{2}{*}{$\begin{array}{l}\text { Estado } \\
\text { Colapso }\end{array}$} \\
\hline 0 & $>=$ & Índice & $<=$ & 0,2 & \\
\hline 0,2 & $>$ & Índice & $<=$ & 0,4 & Crítico \\
\hline 0,4 & $>$ & Índice & $<=$ & 0,6 & Inestable \\
\hline 0,6 & $>$ & Índice & $<=$ & 0,8 & Estable \\
\hline 0,8 & $>$ & Índice & $<=$ & 1 & Óptimo \\
\hline
\end{tabular}

Metodología biógrafa

Fuente: Sepúlveda, $\underline{2008}$ 
El Biograma se fundamenta en el enfoque del desarrollo e integra las dimensiones social y económica, con lo cual permite, finalmente, generar un índice integrado de desarrollo a partir de las tres áreas ${ }^{5}$ :

$I G D=(I S+I E) / 2$

Donde,

IGD es el índice global de desarrollo. Se compone de:
a) IS es el índice social
b) IE es el índice económico

\section{Tipos de gobernanza de las cadenas de valor}

Los pequeños productores agrícolas normalmente están ubicados en segmentos periféricos de las cadenas, y su posición dentro de la estructura de la cadena tiende a estar subordinada a los segmentos más cercanos a los consumidores, ya sean comercializadores o supermercados.

En este sentido, en todas las cadenas de valor existe una gobernanza que determinan las relaciones de poder que se dan entre los diversos actores que las componen. Se pueden hablar de un tipo de gobernanza global de la cadena determinado por el o los actores que ponen las condiciones de poder globales para todos los agentes de la cadena; el segundo tipo de gobernanza se da entre eslabón y eslabón (tipo de gobernanza micro).

En el primer caso, Gereffi clasificó las cadenas según el grado de poder que tenían los agentes dentro de la misma y según las características propias de cada una de acuerdo con las siguientes variables: nivel tecnológico, estructura de control, disponibilidad de recursos, barreras de entrada, entre otras (Gereffi, 1999), por lo tanto, pueden existir dos tipos, una orientada por la oferta y otra que se orienta por la demanda (Gereffi, 1994):

La primera "producer-driven" se encuentra en sectores intensivos en tecnología tales como la industria automotriz, de aviones y de alta tecnología en computadoras. En este sentido, hay fuertes barreras de entrada, pues es necesario contar con altos volúmenes de capital para formar parte de ella.

La otra cadena "buyer -driven" está conformada principalmente por mano de obra, en la que el mercadeo juega un papel de suma importancia. En esta cadena están la industria de la ropa y

\footnotetext{
${ }^{5} \mathrm{~A}$ criterio del investigador, se le otorga el mismo peso relativo a ambas dimensiones pues el objetivo de la investigación es establecer tipologías de las zonas rurales que se vinculan a las agrocadenas, no ahondar sobre el estado de una única dimensión.
}

6

Ivania García Cascante, Jorge Andrey Valenciano Salazar 
los zapatos que son los ejemplos más comunes. Las barreras para entrar a estas cadenas son los costos de inversión para recopilar información del mercado, diseño y desarrollo.

En el segundo caso, la gobernanza que se da entre eslabón y eslabón, existen elementos determinantes a la hora de hacer la clasificación de las cadenas, Gereffi, Humphrey y Sturgeon propusieron cuatro factores específicos para clasificar el tipo de gobierno en las cadenas, dichos factores son los siguientes (Gereffi, Humphrey, \& Sturgeon, 2005, p.85 citado por Díaz y Valenciano, 2012, p.17; Cordero y Valenciano, 2015, p.12):

- La complejidad de la transferencia de la información y el conocimiento requerido para completar una transacción particular, especialmente en las especificaciones de procesos y productos.

- El grado en que la información pueda codificarse y transmitirse, es decir, cómo se transmite eficientemente la información sin necesidad de inversiones por parte de los agentes de la cadena.

- Las capacidades de los actuales y potenciales proveedores en relación con los requerimientos de las transacciones.

- Grado de coordinación explícita y asimetría de poder: se refiere al nivel de claridad que exista en la coordinación en la cadena y a la desigualdad del poder de esta.

Estos tipos de gobernanza son analíticos, no empíricos, aunque, según sus autores, han sido derivados de observaciones empíricas. A continuación se explica en que consiste cada uno de estos tipos de gobernanza (Gereffi, Humphrey, \& Sturgeon, 2005, pp. 83-84; Sturgeon, 2009, pp. 118-119 citado por Díaz y Valenciano, 2012, pp.18-19; Cordero y Valenciano, 2015, p.13):

Coordinación de mercado: acuerdos caracterizados por precios al contado o transacciones repetidas, en las cuales los costos de cambiar los socios son bajos en ambas partes de la cadena. El comprador y el vendedor no necesitan cooperar en la definición del producto, bien porque este se encuentra estandarizado, o porque el proveedor posee suficiente capacidad para proporcionar un resultado satisfactorio. Las transacciones tienen un carácter marcadamente impersonal.

Cadenas de valor modulares: en estas redes industriales los proveedores fabrican productos sujetos a las especificaciones de los clientes, pero con plena autonomía en cuanto a la organización, las competencias y las tecnologías aplicadas. Este tipo de cadenas utilizan maquinaria genérica, de modo que no se encuentran atados a clientes concretos. Este tipo de cadenas son características, por ejemplo en la industria electrónica.

Ivania García Cascante, Jorge Andrey Valenciano Salazar

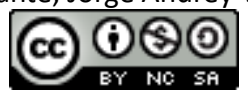

Revista Economía y Sociedad by Universidad Nacional is licensed under a Creative Commons Reconocimiento-NoComercial- 
Cadenas de valor relacionales: cuando no resulta posible codificar todos los aspectos vinculados a las transacciones, surgen redes industriales caracterizadas por interacciones complejas entre compradores y vendedores, quienes mantienen relaciones de cooperación basadas en la reputación y la confianza mutua. Los distritos industriales italianos constituyen un ejemplo de este tipo de cadenas, que son también características, entre otras, de la industria automovilística.

Cadenas de valor cautivas: está presente en las relaciones en donde los pequeños proveedores dependen de uno o varios compradores que por lo general tienen mucho poder. Además, se da un alto control por parte de la empresa líder hacia los proveedores, en donde estos últimos deben de seguir las condiciones que son establecidas por estas empresas que ejercen el poder.

Cadenas de valor jerárquicas: el actor principal de la cadena son empresas trasnacionales, integrada verticalmente con vínculos de control respecto al resto de las empresas que participan en ella mediante relaciones de propiedad.

\section{Situación mundial de las agrocadenas seleccionadas}

Concerniente a la situación mundial de la oferta y de la demanda de estos productos, se observa una marcada dualidad norte-sur, donde los países del norte son los demandantes y además, alojan las casas matrices de las empresas productoras y comercializadoras. Por otro lado, los países del sur (generalmente tropicales) son donde se ubican los procesos productivos de orden agrícola, lo cual se ilustra mediante la tabla 2, según datos al año 2010.

Los países en desarrollo aprovechan sus ventajas comparativas como condiciones climatológicas, suelos, mano de obra, entre otras, para llevar a cabo procesos agrícolas intensivos en tierra y trabajo. De igual forma, se realizan procesos de investigación y desarrollo en donde se aprovecha la cercanía a los centros de producción, esto permite generar mejoras en la calidad de los productos.

Tabla 2.

Principales agentes de las cadenas de café, banano, piña y flores. 2010

\begin{tabular}{c|c|c|c|c}
\hline PAÍSES & PIÑA & BANANO & CAFÉ & FLORES \\
\hline & Costa Rica & Ecuador & Brasil & Holanda \\
Principales países & Bélgica & Bélgica & Vietnam & Colombia \\
productores para el & Colombia & Colombia & Ecuador \\
mercado mundial & Ecuador & Costa Rica & & Kenya \\
\hline
\end{tabular}

8

Ivania García Cascante, Jorge Andrey Valenciano Salazar

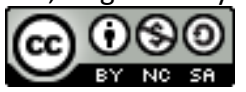

Revista Economía y Sociedad by Universidad Nacional is licensed under a Creative Commons Reconocimiento-NoComercial- 


\begin{tabular}{|c|c|c|c|c|}
\hline PAÍSES & PIÑA & BANANO & CAFÉ & FLORES \\
\hline $\begin{array}{l}\text { Principales países } \\
\text { consumidores }\end{array}$ & $\begin{array}{c}\text { Estados Unidos } \\
\text { Bélgica } \\
\text { Alemania } \\
\text { Holanda } \\
\text { Reino Unido } \\
\end{array}$ & $\begin{array}{l}\text { Estados Unidos } \\
\text { Bélgica } \\
\text { Alemania } \\
\text { Japón } \\
\text { Reino Unido } \\
\end{array}$ & $\begin{array}{l}\text { Estados Unidos } \\
\text { Bélgica } \\
\text { Japón }\end{array}$ & $\begin{array}{c}\text { Holanda } \\
\text { Alemania } \\
\text { Reino Unido } \\
\text { Estados Unidos }\end{array}$ \\
\hline Empresas productoras & \multicolumn{2}{|c|}{$\begin{array}{l}\text { Chiquita } \\
\text { Dole } \\
\text { Del monte } \\
\text { Fyffes }\end{array}$} & $\begin{array}{c}\text { Neumann Kaffee } \\
\text { Gruppe, Volcafe, } \\
\text { and Ecom } \\
\text { Agroindustrial }\end{array}$ & $\begin{array}{c}\text { Fides B.V } \\
\text { Syngenta Flowers } \\
\text { Beekenkamp } \\
\text { Selecta Klemm } \\
\text { Dummen } \\
\text { BallF LoraPlant } \\
\text { ForemostCo } \\
\text { Cohen Nurseries } \\
\text { Oro Farms } \\
\text { Mc Gregor Plant } \\
\text { Sales/Florexpo }\end{array}$ \\
\hline
\end{tabular}

Fuente: elaboración propia a partir de García (2012).

\subsection{Las agrocadenas en los medios rurales}

El elemento diferenciador en este caso entre urbano y rural está dado por el uso de la tierra en función del tipo de actividades económicas que se desarrollan en cada territorio.

Esta perspectiva llevaría al análisis de las dinámicas que condicionan el cambio de uso de la tierra, desde actividades primarias hacia actividades productivas relacionadas con aglomeración de la población y la correspondiente urbanización. En este caso las situaciones extremas son lo rural como sinónimo de uso de la tierra en actividades primarias y lo urbano como sinónimo de uso de la tierra en actividades fundamentalmente de servicios para una población altamente aglomerada. (Rodríguez. A; Saborío, M; 2007, p.15).

Las cuatro agrocadenas de estudio se caracterizan por realizar los procesos productivos en localidades rurales, las cuales cuentan con las condiciones necesarias para producir bienes de calidad de exportación. Esta situación se resume en la tabla 3.

Tabla 3.

Costa Rica: Distritos con presencia de IED en la producción de café, banano, piña y flores. 2010.

\begin{tabular}{l|l|l|l}
\hline Agrocadena & \multicolumn{1}{|c|}{$\begin{array}{c}\text { Productos en la } \\
\text { agrocadena }\end{array}$} & \multicolumn{1}{|c}{ Comunidades involucradas } & \multicolumn{1}{c}{$\begin{array}{c}\text { Transnacional } \\
\text { involucrada }\end{array}$} \\
\hline Piña & $\begin{array}{l}\text { Producto fresco } \\
\text { Jugos y purés de } \\
\text { frutas }\end{array}$ & $\begin{array}{l}\text { Guácimo, Siquirres, Matina, Los } \\
\text { Chiles, San Carlos, Sarapiquí, } \\
\text { Guatuso, Buenos Aires, Parrita, Pérez } \\
\text { Zeledón y Upala }\end{array}$ & $\begin{array}{l}\text { Del Monte, Chiquita } \\
\text { Brands y Dole }\end{array}$ \\
\hline
\end{tabular}

Ivania García Cascante, Jorge Andrey Valenciano Salazar

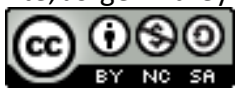

Revista Economía y Sociedad by Universidad Nacional is licensed under a Creative Commons Reconocimiento-NoComercial- 


\begin{tabular}{l|l|l|l}
\hline \multicolumn{1}{|c|}{ Agrocadena } & \multicolumn{1}{|c|}{$\begin{array}{c}\text { Productos en la } \\
\text { agrocadena }\end{array}$} & \multicolumn{1}{|c}{ Comunidades involucradas } & \multicolumn{1}{c}{$\begin{array}{c}\text { Transnacional } \\
\text { involucrada }\end{array}$} \\
\hline Banano & $\begin{array}{l}\text { Producto fresco } \\
\text { Jugos y purés de } \\
\text { frutas }\end{array}$ & $\begin{array}{l}\text { Matina, Pococí, Siquirres, Parrita y } \\
\text { Corredores }\end{array}$ & $\begin{array}{l}\text { Del Monte, Chiquita } \\
\text { Brands y Dole }\end{array}$ \\
\hline Café & Café oro & $\begin{array}{l}\text { Tarrazú, Dota, Naranjo, Alajuela, } \\
\text { León Cortés }\end{array}$ & $\begin{array}{l}\text { Volcafé, Neuman Kaffee } \\
\text { Group, Ecom }\end{array}$ \\
\hline Flores & Flores & $\begin{array}{l}\text { Paraíso, San José de la Montaña, } \\
\text { Barva }\end{array}$ & $\begin{array}{l}\text { Expoflora, Florexpo, } \\
\text { Corifresco }\end{array}$ \\
\hline
\end{tabular}

Fuente: García, 2012.

La piña es un bien tradicionalmente producido en Costa Rica, sin embargo, la producción intensiva con utilización de un paquete tecnológico sofisticado inició hasta los años setenta, con la participación de PINDECO ${ }^{6}$, subsidiaria de Del Monte, en Buenos Aires de Puntarenas. La producción piñera nacional se centraliza en la Zona Norte, Sur y Caribe. En la Zona Norte, el cultivo de piña se asienta en los distritos de Río Cuarto, Aguas Zarcas, Valencia, Pital y Upala; en los cuales, los pequeños y medianos productores poseen una gran participación. En Zona Norte, la producción piñera ha experimentado un crecimiento acelerado dado que en el año 1999 el $41,6 \%$ del total de hectáreas sembradas se destinaban a la producción de piña, mientras que en el 2004 este porcentaje se incrementó a un $52 \%$.

En la Zona Sur la producción piñera se concentra en los distritos de Volcán y Buenos Aires, en manos de la transnacional PINDECO, restándoles protagonismo a los productores independientes. Y por último, la producción de piña en la Zona del Caribe comprende los distritos de Rita, Roxana, Pacuarito, Guácimo, Pocora, Rio Jiménez, y Duacari; y se encuentra, casi en su totalidad, en manos de empresas transnacionales. Asimismo, se han incrementado las hectáreas destinadas a este cultivo al pasar de 6,1 \% en 1999 a 21,7 \% en el 2004.

Por su parte, la producción bananera se caracteriza por desarrollarse principalmente en la zona Atlántica, más del $90 \%$ de las exportaciones provienen de la zona. Finalmente, según el censo cafetalero 2006, el 62 \% del uso del suelo se destinaba a café en el cantón de Tarrazú, en León Cortés era del $65 \%$ y en Dota representaba el $49 \%$ del uso del suelo (García, 2012). En el caso de flores, la producción se concentró en San José de la Montaña, Paraíso y Barva.

\subsection{Estructura productiva de las agrocadenas en los espacios rurales}

Con el fin de caracterizar la dinámica productiva de los espacios rurales, a continuación se resume los principales vínculos productivos y de comercialización entre los diferentes actores de dichos espacios (García, 2012):

${ }^{6}$ Pineapple Development Corporation-Del Monte.

10

Ivania García Cascante, Jorge Andrey Valenciano Salazar

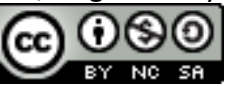

Revista Economía y Sociedad by Universidad Nacional is licensed under a Creative Commons Reconocimiento-NoComercial- 
- Grandes grupos transnacionales (GGT): son empresas de capital extranjero, cuya casa matriz se encuentra en países desarrollados y cuentan con divisiones o subsidiarias en los países subdesarrollados, los cuales abastecen la cadena del producto principal. Estas empresas poseen activos en el territorio nacional, tales como maquinaria, equipo, instalaciones y tierra; además cuentan con procesos de producción y logística bien definidos. Estos grupos transnacionales se abastecen del producto principal de tres formas: (i) lo producen en sus propias fincas para lo que emplean fuerza de trabajo local; (ii) lo compran a productores independientes; (iii) lo compran a grupos organizados tales como cooperativas de productores.

- Productores independientes (PI): este grupo produce las frutas o el café en sus fincas independientes que son propiedad familiar. Para vender su producto a las grandes transnacionales deben cumplir con todos los requisitos que estas exijan tales como calidad del producto, legalidad con los trabajadores, entregas, entre otros aspectos. En el café es más común encontrar productores independientes que en el caso de las frutas.

- Cooperativas: en todos los productos existen diferentes grupos cooperativos que reúnen a algunos productores y les brindan ciertos beneficios. Estas cooperativas pueden exportar directamente al mercado internacional, sin embargo, es más frecuente que le vendan su producción a los Grandes Grupos y Transnacionales. En el café el número de cooperativas es superior al de los otros bienes, dado que existe un contexto histórico que potencializa la organización, lo que ha potenciado que en el caso del café la propiedad de la tierra pertenece a los productores y no a los grupos transnacionales como en el caso de la piña y el banano. Según datos de Promotora de Comercio Exterior de Costa Rica (PROCOMER) en el 2010 existían 15 cooperativas exportadoras de café, mientras que en banano y piña eran solo 3 y 4 respectivamente.

- Grupos empresariales de capital extranjero (GECE): estos son grandes empresas de capital mixto o de extranjeros que viven permanentemente en el país. Se caracterizan por que la familia y los activos del inversionista foráneo se establece en el país con lo que toda o la mayor parte de la utilidades se mantiene en las fronteras nacionales. En el caso de la piña y el banano se puede mencionar como ejemplo el Grupo Acon que es de capital chino establecida en el Atlántico y que posee grandes plantaciones, otro ejemplo es el Beneficio La Eva que pertenece a italianos, en la localidad de Sarchí y está en el país desde el 2005.

\subsection{Gobernanza en los productos analizados}

Al analizar el tipo de gobernanza macro de cada una de las cadenas bajo análisis se concluye las cadenas de los cuatro productos están orientadas por la demanda, ya que es de suma importancia para el comprador aspectos como la calidad y las certificaciones mundiales (García, 2012). Sin embargo, a partir del enfoque de las cadenas de valor, focalizando en el nivel inter- 
segmentos de la cadena, que rige la relación de los productores agrícolas con el siguiente eslabón de la cadena (gobernanza micro), se pueden precisar otros tipos de gobernanza en cada cadena bajo en estudio.

En los casos de la piña y el banano, la gobernanza es cautiva ya que las multinacionales tienen plantaciones propias; no obstante, también establecen contratos con pequeños productores que comprenden y aceptan una serie de estatutos (confidencialidad, calidad, entre otros) que garanticen que el producto se asemeje al que las grandes compañías producen en sus propias fincas. En la etapa de exportación el control es jerárquico, pues el proceso de logística es controlado por la compañía multinacional, ya que cuenta con toda la maquinaria, el conocimiento del proceso y los recursos económicos necesarios.

En cuanto al café, hay una gobernanza cautiva entre la producción y el beneficiado, hay un grupo cuantioso de pequeños productores independientes o constituidos en cooperativas los cuales les venden a las mismas cooperativas o beneficios de café privados. En la exportación la relación tiende a ser jerárquica pues las transnacionales cuentan con sus propias empresas que se encargan del beneficiado y la exportación del grano.

En la producción de flores cada empresa se encarga del proceso, tanto de importar semillas como de cultivar y sembrar la flor. Hay ausencia de compras a productores independientes. En empresas grandes también se encargan de todo el proceso de exportación, sin embargo el comprador se encarga de llevar al destino final las flores; en estos casos la gobernanza tiende a ser modular.

\section{Estado del desarrollo en los espacios rurales}

\subsection{Aproximación del Índice General de Desarrollo Humano (IGDH)}

Con el índice calculado sobre desarrollo humano, se observa que de los 52 distritos que representan los cuatro productos de estudio, únicamente 6 de ellos se encontraban entre los primeros 100 lugares según los datos utilizados del censo 2000 y únicamente 5 distritos según los datos del censo 2011 se encontraban entre los primeros 100 lugares (ver anexo 1).

Los distritos de Barva, Grecia y San Ramón productores de flores y café, respectivamente, están en los primeros lugares en desarrollo humano del 2011, sin embargo hay un descenso en la posición de estos distritos con respecto al año 2000. Se destaca la situación de San José de la Montaña y Aguas Calientes de Cartago, ambos distritos productores de flores, y que ascendieron varias posiciones en el año 2011.

12

Ivania García Cascante, Jorge Andrey Valenciano Salazar

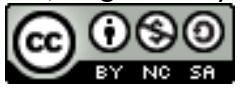

Revista Economía y Sociedad by Universidad Nacional is licensed under a Creative Commons Reconocimiento-NoComercial- 
En un balance general, se tiene que de los 52 distritos analizados 35 desmejoraron su posición en el IGDH del 2000 al 2011 lo que representan un 67\%, finalmente 16 mejoraron (31 $\%)$ y uno se mantuvo igual (2\%).

En el caso de Barva de Heredia, el IGDH en el 2000 tuvo 0.76 y en el 2011 fue de $0.80^{7}$. Se podría pensar que la situación fue favorable, pero esto no es correcto pues los valores de medición de los máximos y mínimos de cada año son distintos. Lo apropiado es notar que en el 2000 Barva ocupaba la posición 20 de 460 distritos, mientras que en el 2011 descendió a la posición 26, lo cual significa más bien que su situación empeoró en forma relativa a otros distritos.

Llaman la atención caídas abruptas en el IGDH en distritos como Buenos Aires de Puntarenas, que pasó de la posición 236 a la 357 del año 2000 al 2011, y la de Carrandí de Matina, que pasó de la posición 310 a la 417, para el mismo periodo. Situación contraria se refleja en los distritos de General de Pérez Zeledón y Jardín de Dota, los cuales fueron los distritos de mayor acenso, escalando más de 80 posiciones durante los años analizados.

La tabla 4 muestra como del 2000 al 2011 aumenta la concentración de distritos analizados en posiciones intermedias del Índice Global de Desarrollo Humano. En el año 2000 solamente el 52 \% de los distritos tenían un IGDH de entre 0,51 a 0,75 mientras que en el año 2011 ese porcentaje creció hasta el $75 \%$ de los distritos en ese rango del índice. Por otro lado, baja la cantidad de distritos ubicados en el rango más bajo del índice.

Tabla 4.

Costa Rica: cantidad de distritos según posicionamiento en el Índice Global de Desarrollo Humano según datos de los censos de los siguientes periodos 2000 y 2011

\begin{tabular}{c|cc|cc}
\hline \multirow{2}{*}{ Rango del Índice Global } & \multicolumn{4}{|c}{ Cantidad de distritos } \\
\cline { 2 - 5 } & \multicolumn{2}{|c}{$\mathbf{2 0 0 0}$} & \multicolumn{2}{c}{$\mathbf{2 0 1 1}$} \\
\hline $\mathbf{0 , 2 5 - 0 , 5 0}$ & Absoluto & $\%$ & Absoluto & $\%$ \\
$\mathbf{0 , 5 1 - 0 , 7 5}$ & 24 & $46,15 \%$ & 12 & $23,08 \%$ \\
$\mathbf{0 , 7 6 - 1}$ & 27 & $51,92 \%$ & 39 & $75,00 \%$ \\
TOTAL & 1 & $1,92 \%$ & 1 & $1,92 \%$ \\
& 52 & $100,00 \%$ & 52 & $100,00 \%$ \\
\hline
\end{tabular}

Fuente: elaboración propia con datos del INEC, censos $\underline{2000}$ y 2011

\footnotetext{
${ }^{7}$ Se debe tener cautela con la interpretación de los índices, pues estos están construidos a partir de la fórmula de reescalamiento, procedimiento que utiliza los valores máximos y mínimos de cada año, valores que no son estáticos. Una ventaja de ellos, sin embargo, es que se puede medir el desarrollo de los distritos con respecto al desarrollo que tienen sus homólogos del resto del país y no solo con respecto a sí mismos.
} 


\subsection{Hacia una búsqueda de tipologías}

Con el fin de aproximar una tipología a partir del desempeño del desarrollo local, se analizan individualmente los componentes del IGDH (índice social e índice económico).

El índice económico (IE) presenta el siguiente balance: 23 distritos mejoraron su posición y 29 desmejoraron. Entre los distritos que ascendieron más posiciones están General de Pérez Zeledón, Cariari y Rita de Pococí. Entretanto, los distritos de Platanares de Pérez Zeledón, Buenos Aires y Río Cuarto fueron los que experimentaron mayores descensos en este índice.

El balance general del Índice Social (IS) es el siguiente: 23 distritos mejoraron su posición, 27 desmejoraron y 2 se mantuvieron sin variación. Los descensos más importante los experimentaron Buenos Aires, Batán y Carrandí que cayeron más de 100 posiciones, mientras que los principales ascensos fueron Platanares de Pérez Zeledón y Jardín de Dota.

La tabla 5 resume los principales resultados del IS y el IE: solo $12 \%$ de los distritos escalaron de posición tanto en lo económico como en lo social, mientras que 19 \% descendieron en ambos índices. En 33 \% de los distritos hubo ascensos en la posición del IE, pero descensos en el IS y viceversa, mientras que $4 \%$ descendieron en el IE y se mantuvieron en la misma posición del IS.

Tabla 5.

Costa Rica: Balance general del Índice Social y del Índice Económico del 2000 al 2011

\begin{tabular}{lccc}
\hline \multicolumn{1}{c}{ Social } & Económico & Total de distritos & Porcentaje \\
\hline Mejoró & Mejoró & 6 & $12 \%$ \\
\hline Empeoró & Mejoró & 17 & $33 \%$ \\
\hline Se mantiene & Mejoró & 0 & $0 \%$ \\
\hline Mejoró & Empeoró & 17 & $33 \%$ \\
\hline Empeoró & Empeoró & 10 & $19 \%$ \\
\hline Se mantiene & Empeoró & 2 & $4 \%$ \\
\hline
\end{tabular}

Fuente: elaboración propia con datos del INEC, censos 2000 y 2011

Con el fin de establecer un esquema que permita identificar tipologías de desarrollo se presenta una clasificación del IE y el IS a partir de las categorías del Biograma. Para ello se establecen siete tipologías mediante la combinación del desempeño del IE y el IS, así como una distribución por producto en cada distrito utilizando el índice del año 2011. La tabla 6 presenta los distritos según la tipología utilizada en cada uno de los casos.

14 
Tabla 6.

Costa Rica: clasificación de los distritos según tipología y producto para el año 2011

\begin{tabular}{|c|c|c|c|c|c|c|c|}
\hline & Tipo I & Tipo II & Tipo III & Tipo IV & Tipo V & Tipo VI & Tipo VII \\
\hline Café & $\begin{array}{l}\text { Orosi } \\
\text { Copey } \\
\text { Jardín } \\
\text { Santa María } \\
\text { San Antonio } \\
\text { San Isidro } \\
\text { San Pablo } \\
\text { Santa Cruz } \\
\text { Daniel Flores } \\
\text { General } \\
\end{array}$ & $\begin{array}{l}\text { Llano Bonito } \\
\text { San Andrés } \\
\text { Cajón } \\
\text { Páramo } \\
\text { Pejibaye } \\
\text { Platanares } \\
\text { Río Nuevo } \\
\text { Rivas } \\
\text { San Pedro }\end{array}$ & & $\begin{array}{l}\text { Grecia } \\
\text { Palmares } \\
\text { San Ramón } \\
\text { San Isidro } \\
\text { del General }\end{array}$ & & & \\
\hline Banano & $\begin{array}{l}\text { Batán } \\
\text { Siquirres }\end{array}$ & Mercedes & $\begin{array}{l}\text { Carrandí } \\
\text { Matina } \\
\text { Colorado }\end{array}$ & & & $\begin{array}{l}\text { Valle La } \\
\text { Estrella }\end{array}$ & \\
\hline Piña & $\begin{array}{l}\text { Aguas Zarcas } \\
\text { Venecia } \\
\text { Pocora }\end{array}$ & $\begin{array}{l}\text { Río Cuarto } \\
\text { Upala } \\
\text { Buenos Aires } \\
\text { Volcán }\end{array}$ & Pacuarito & & & & Pital \\
\hline Flores & Guápiles & & & $\begin{array}{l}\text { Turrialba } \\
\text { Jesús }\end{array}$ & $\begin{array}{l}\text { Aguacaliente } \\
\text { o San Fco. } \\
\text { Barva } \\
\text { San José de } \\
\text { la Montaña }\end{array}$ & & \\
\hline $\begin{array}{l}\text { Banano } \\
\text { y piña }\end{array}$ & $\begin{array}{l}\text { Guácimo } \\
\text { Cariari }\end{array}$ & Duacarí & $\begin{array}{l}\text { Río Jiménez } \\
\text { Rita } \\
\text { Roxana }\end{array}$ & & & & \\
\hline $\begin{array}{l}\text { Café/ } \\
\text { Flor }\end{array}$ & Santiago & & & & & & \\
\hline
\end{tabular}

Fuente: elaboración propia con base en índices calculaos de desarrollo económico y social

El significado de cada una de las tipologías se explica a continuación:

- Tipo I: se caracteriza por un desarrollo económico inestable y un desarrollo social estable, es la más común entre los distritos estudiados. La mayoría de los distritos (52,6 $\%)$ en este grupo producen café, pero también están en menor medida otros productos como piña y banano

- Tipo II: se caracteriza por un desarrollo económico crítico y un desarrollo social estable. La mayoría de los distritos en este grupo producen café (60 \%) y piña $(26,7 \%)$. 
- Tipo III: es una de las menos deseadas pues el desarrollo económico es crítico y el social es inestable. Se compone de distritos de la zona Atlántica que producen piña y banano.

- Tipo IV: se caracteriza por un desarrollo económico inestable y un desarrollo social óptimo. Se compone de distritos productores de flores (33 \%) y café (67\%).

- Tipo V: es la de mejor desempeño, pues el desarrollo económico es estable y el social óptimo. Son productores de flores.

- Tipo VI: es el de peor desempeño con desarrollo social y económico crítico. Solamente está el distrito de Valle de la Estrella.

- Tipo VII: tiene un desempeño deficiente con desarrollo social y económico inestable. Solo está el distrito de Pital de San Carlos.

Tabla 7.

Clasificación de tipologías de los distritos, según los productos analizados en el 2011

\begin{tabular}{|c|c|c|c|c|c|c|c|c|c|c|c|c|c|c|}
\hline \multirow[b]{2}{*}{ Producto } & \multicolumn{2}{|c|}{ Tipo I } & \multicolumn{2}{|c|}{ Tipo II } & \multicolumn{2}{|c|}{ Tipo III } & \multicolumn{2}{|c|}{ Tipo IV } & \multicolumn{2}{|c|}{ Tipo V } & \multicolumn{2}{|c|}{ Tipo VI } & \multicolumn{2}{|c|}{ Tipo VII } \\
\hline & Total & $\%$ & Total & $\%$ & Total & $\%$ & Total & $\%$ & Total & $\%$ & Total & $\%$ & Total & $\%$ \\
\hline Café & 10 & 52,6 & 9 & 60,0 & 0 & 0,0 & 4 & 66,7 & 0 & 0 & 0 & 0 & 0 & 0 \\
\hline Banano & 2 & 10,5 & 1 & 6,7 & 3 & 42,9 & 0 & 0,0 & 0 & 0 & 1 & 100 & 0 & 0 \\
\hline Piña & 3 & 15,8 & 4 & 26,7 & 1 & 14,3 & 0 & 0,0 & 0 & 0 & 0 & 0 & 1 & 100 \\
\hline Flores & 1 & 5,3 & 0 & 0,0 & 0 & 0,0 & 2 & 33,3 & 3 & 100 & 0 & 0 & 0 & 0 \\
\hline $\begin{array}{l}\text { Banano y } \\
\text { piña }\end{array}$ & 2 & 10,5 & 1 & 6,7 & 3 & 42,9 & 0 & 0,0 & 0 & 0 & 0 & 0 & 0 & 0 \\
\hline Café/Flor & 1 & 5,3 & 0 & 0,0 & 0 & 0,0 & 0 & 0,0 & 0 & 0 & 0 & 0 & 0 & 0 \\
\hline TOTAL & 19 & 100 & 15 & 100 & 7 & 100 & 6 & 100 & 3 & 100 & 1 & 100 & 1 & 100 \\
\hline
\end{tabular}

Fuente: elaboración propia con datos del INEC, censos $\underline{2000}$ y $\underline{2011}$

\section{Conclusiones}

La mayoría de distritos analizados desmejoraron su posición dentro del índice global de desarrollo humano calculado IGDH, según los datos utilizados de los censos 2000 y 2011, el $67 \%$ de los distritos donde hay presencia de IED en actividades agrícolas desmejoraron su situación.

Se diferencian siete tipos de desarrollo humano de los distritos receptores de IED, la mayoría de distritos están en el tipo I, con un desarrollo económico inestable y socialmente estable, y el tipo II que es crítico y estable, respectivamente.

De acuerdo con los efectos esperados de la IED, se desprende que para los productos estudiados hay un crecimiento económico, basándose en que el $45 \%$ de los distritos mejoro su posición en el índice económico; sin embargo, este crecimiento no debe de confundirse con

16

Ivania García Cascante, Jorge Andrey Valenciano Salazar

\section{(c) (i)(2)}

Revista Economía y Sociedad by Universidad Nacional is licensed under a Creative Commons Reconocimiento-NoComercial- 
desarrollo, dado que su esperado efecto derrame pareciera no haber ocurrido, lo cual puede estar explicado por el control que realizan las empresas más fuertes sobre las agrocadenas, lo cual les permite el control de precios y establecimiento de condiciones de producción.

Los distritos de mayor descenso en el IGDH son productores de banano y piña y los distritos de mayor ascenso son productores de café y flores; sin dejar de señalar que históricamente la estructura productiva del café se orienta más a productores familiares dueños de plantaciones. Esta es una diferencia sustancial, si se compara con el hecho de que en las frutas la tendencia se orienta más a emplear mano de obra no calificada, por parte de una transnacional, basta con ver el número de cooperativas de café, que superan las de otros productos.

Además, se logró establecer dos tipologías de desarrollo, para los distritos receptores de la IED. El tipo I tiene un desarrollo económico inestable y un desarrollo social estable. El tipo II tiene ambos índices estables. Las localidades donde operan las agrocadenas de piña y banano se mantuvieron en el tipo I durante toda la década, mientras que el café avanzó al Tipo II.

Además, se discernió entre cuatro actores generales en los espacios rurales de las cadenas de productos agrícolas. El primer grupo lo conforman los pequeños productores independientes, el segundo son los productores organizados, como es el caso de las cooperativas o asociaciones locales, el tercero son las grandes empresas de capital nacional las cuales compiten con las compañías extranjeras, por último, están las empresas transnacionales las cuales tienen mayor capital y presencia en el mercado nacional e internacional.

\section{Referencias}

Cordero, R., Valenciano, J. (2015). Gobernanza de las empresas dominantes en la cadena de café: el caso de la región de Turrialba, Costa Rica. Revisa ABRA, (35)50, 1-26. doi: http://dx.doi.org/10.15359/abra.35-51.9

Cubero, R. (2006). Determinantes y efectos económicos de la Inversión Extranjera Directa: Teoría y evidencia internacional. En López, G. y Umaña, C. (Eds.), Inversión extranjera en $\begin{array}{llll}\text { Centroamérica } & \text { (pp. 1-51). } & \text { Recuperado de }\end{array}$ http://www19.iadb.org/intal/intalcdi/PE/2009/02595.pdf

Díaz, R., Valenciano, J. (2011). Inversión extranjera directa y desarrollo sostenible en espacios rurales. Revista Comercio Exterior, (61)5, 1-48. Recuperado de http://revistas.bancomext.gob.mx/rce/magazines/147/1/RCE PDF VOL. 615 SEPTIEMBRE_OCTUBRE_2011.pdf 
Díaz, R., Valenciano, J. (2012) Gobernanza en las cadenas globales de mercancías/valor: una revisión conceptual. Revista Economía y Sociedad, (17)41, 9-27. Recuperado de http://www.revistas.una.ac.cr/index.php/economia/article/view/4906

Gallagher, K., \& López, A. (2008). Resumen ejecutivo. En Gallagher, K, Porzecanski, R., \& López, A. (Eds.), Inversión Extranjera Directa y Desarrollo Sustentable: Lecciones desde las Américas (pp.VII-XI). Recuperado de https://ase.tufts.edu/gdae/Pubs/rp/FDI_WG_May08_Span_Full.pdf

García, I. (2012). Modalidades de la Inversión Extranjera Directa en las Cadenas Agroindustriales de Costa Rica: Efectos en los Medios Rurales (Tesis de Licenciatura). Universidad Nacional, Heredia, Costa Rica.

Gereffi, G. (1994). The Organization of Buyer-Driven Global Commodity Chains: How U.S. Retailers Shape Overseas Production Networks. En Gereffi, G. y Korzeniewicz, M (Eds), Commodity Chains and Global Capitalism (pp.93-95). Recuperado de https://books.google.co.cr/books?id=A86j9pWfTcAC\&printsec=frontcover\&hl=es\&sourc e=gbs ge summary $r \& c a d=0 \# v=0 n e p a g e \& q \& f=f a l s e$

Gereffi, G. (1999). International Trade and industrial upgrading in the apparel commodity chain. Journal of International Economics. 48(1), 37-99. doi: http://dx.doi.org/10.1016/S0022$\underline{1996(98) 00075-0}$

Gereffi, G.; Humphrey, J., \& Sturgeon, T. (2005). The Governance of Global value chains. Review of International Political Economy. 12(1), 78-104. doi: http://dx.doi.org/10.1080/09692290500049805

Instituto Nacional de Estadística y Censo. (2000). Censo Nacional de Población 2000. Recuperado de http://www.inec.go.cr/

Instituto Nacional de Estadística y Censo. (2011). Censo Nacional de Población 2011. Recuperado de http://www.inec.go.cr/

Rodríguez. A; Saborío, M. (Eds.). (2007). Lo rural es diverso: Evidencia para el caso de Costa Rica. Recuperado de http://repiica.iica.int/docs/B0706E/B0706E.PDF

Sepúlveda, S. (2008). Biograma: Metodología para estimar el desarrollo sostenible de territorios. Recuperado de http://observatorio.ruralytierras.gob.bo/menu/principal/doc/BIOGRAMA\%202008.pdf

18

Ivania García Cascante, Jorge Andrey Valenciano Salazar

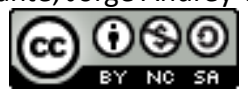

Revista Economía y Sociedad by Universidad Nacional is licensed under a Creative Commons Reconocimiento-NoComercialCompartirlgual 4.0 Internacional License. Creado a partir de la obra en http://www.revistas.una.ac.cr/index.php/economia 
Sturgeon, T. (2009). Frontiers of Commodity Chain Research: Interdisciplinary theory building in an age of globalization. En Bair, J (Ed), Frontiers of Commodity Chain Research (pp. 110134). California, Stanford University Press. 
Anexo 1.

Índice Global de Desarrollo Humano de Costa Rica 2000 y 2011 según distritos con presencia de IED

\begin{tabular}{|c|c|c|c|c|c|c|c|c|}
\hline PRODUCTO & PROVINCIA & CANTÓN & DISTRITO & $\begin{array}{c}\text { ÍNDICE } \\
\text { GLOBAL } \\
2000 \\
\end{array}$ & $\begin{array}{c}\text { ÍNDICE } \\
\text { GLOBAL } \\
2011 \\
\end{array}$ & $\begin{array}{l}\text { RANK } \\
2000\end{array}$ & $\begin{array}{c}\text { RANK } \\
2011\end{array}$ & $\begin{array}{c}\text { POSICION } \\
\text { ASCENSO } \\
\text { /DESCENSO } \\
\end{array}$ \\
\hline Flor & Heredia & Barva & Barva & 0,76 & 0,80 & 20 & 26 & -6 \\
\hline Café & Alajuela & Grecia & Grecia & 0,70 & 0,75 & 57 & 64 & -7 \\
\hline Café & Alajuela & San Ramón & San Ramón & 0,69 & 0,75 & 58 & 68 & -10 \\
\hline Flor & Heredia & Barva & San José de la Montaña & 0,64 & 0,75 & 122 & 77 & 45 \\
\hline Flor & Cartago & Cartago & Aguacaliente o San Fco. & 0,66 & 0,74 & 95 & 79 & 16 \\
\hline Café & Alajuela & Palmares & Palmares & 0,66 & 0,72 & 89 & 105 & -16 \\
\hline Flor & Heredia & Santa Bárbara & Jesús & 0,65 & 0,72 & 108 & 111 & -3 \\
\hline Flor & Cartago & Turrialba & Turrialba & 0,61 & 0,71 & 156 & 119 & 37 \\
\hline Café & San José & Pérez Zeledón & San Isidro del General & 0,61 & 0,68 & 169 & 148 & 21 \\
\hline Flor & Limón & Pococí & Guápiles & 0,62 & 0,68 & 143 & 149 & -6 \\
\hline Café & San José & Dota & Santa María & 0,62 & 0,68 & 142 & 150 & -8 \\
\hline Café & San José & Pérez Zeledón & Daniel Flores & 0,57 & 0,65 & 218 & 191 & 27 \\
\hline Café & Cartago & Paraíso & Orosi & 0,58 & 0,64 & 207 & 209 & -2 \\
\hline Café & San José & Pérez Zeledón & General & 0,51 & 0,64 & 301 & 217 & 84 \\
\hline Café & San José & León Cortés & San Pablo & 0,57 & 0,63 & 226 & 221 & 5 \\
\hline Café & San José & León Cortés & San Antonio & 0,56 & 0,63 & 237 & 222 & 15 \\
\hline Piña & Alajuela & San Carlos & Venecia & 0,56 & 0,63 & 232 & 230 & 2 \\
\hline Piña & Alajuela & San Carlos & Aguas Zarcas & 0,54 & 0,62 & 262 & 251 & 11 \\
\hline Piña/banano & Limón & Guácimo & Guácimo & 0,55 & 0,61 & 244 & 266 & -22 \\
\hline Banano & Limón & Guácimo & Mercedes & 0,54 & 0,60 & 256 & 272 & -16 \\
\hline Café/flor & Cartago & Paraíso & Santiago & 0,55 & 0,60 & 247 & 284 & -37 \\
\hline Café & San José & León Cortés & San Andrés & 0,54 & 0,59 & 267 & 292 & -25 \\
\hline Banano & Limón & Siquirres & Siquirres & 0,53 & 0,59 & 278 & 293 & -15 \\
\hline Piña & Limón & Guácimo & Pocora & 0,53 & 0,59 & 272 & 301 & -29 \\
\hline Café & San José & León Cortés & San Isidro & 0,53 & 0,58 & 269 & 308 & -39 \\
\hline Café & San José & Dota & Jardín & 0,44 & 0,57 & 394 & 314 & 80 \\
\hline Café & San José & Pérez Zeledón & Rivas & 0,49 & 0,57 & 329 & 315 & 14 \\
\hline Café & San José & Dota & Copey & 0,53 & 0,57 & 281 & 319 & -38 \\
\hline Café & San José & Pérez Zeledón & Páramo & 0,47 & 0,56 & 360 & 328 & 32 \\
\hline Café & San José & León Cortés & Santa Cruz & 0,49 & 0,56 & 321 & 329 & -8 \\
\hline Piña/banano & Limón & Pococí & Cariari & 0,48 & 0,55 & 346 & 337 & 9 \\
\hline Café & San José & León Cortés & Llano Bonito & 0,50 & 0,55 & 317 & 347 & -30 \\
\hline Piña & Puntarenas & Buenos Aires & Buenos Aires & 0,56 & 0,54 & 236 & 357 & -121 \\
\hline Café & San José & Pérez Zeledón & Cajón & 0,48 & 0,54 & 342 & 358 & -16 \\
\hline Piña/banano & Limón & Guácimo & Duacarí & 0,46 & 0,54 & 368 & 362 & 6 \\
\hline Café & San José & Pérez Zeledón & Platanares & 0,47 & 0,54 & 348 & 363 & -15 \\
\hline Banano & Limón & Matina & Batán & 0,49 & 0,53 & 322 & 366 & -44 \\
\hline Piña & Alajuela & Grecia & Río Cuarto & 0,52 & 0,53 & 292 & 373 & -81 \\
\hline Piña & Alajuela & Upala & Upala & 0,47 & 0,53 & 362 & 375 & -13 \\
\hline
\end{tabular}

20

Ivania García Cascante, Jorge Andrey Valenciano Salazar

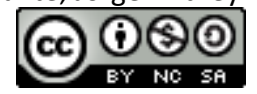

Revista Economía y Sociedad by Universidad Nacional is licensed under a Creative Commons Reconocimiento-NoComercialCompartirlgual 4.0 Internacional License.

Creado a partir de la obra en http://www.revistas.una.ac.cr/index.php/economia 
Economía y Sociedad, Vol. 21, № 49, enero-junio 2016, pp. 1-21

EISSN: 2215-3403 URL http://www.revistas.una.ac.cr/economia

Economía QSOCIEDAD

\begin{tabular}{|c|c|c|c|c|c|c|c|c|}
\hline PRODUCTO & PROVINCIA & CANTÓN & DISTRITO & $\begin{array}{c}\text { ÍNDICE } \\
\text { GLOBAL } \\
2000 \\
\end{array}$ & $\begin{array}{c}\text { ÍNDICE } \\
\text { GLOBAL } \\
2011 \\
\end{array}$ & $\begin{array}{c}\text { RANK } \\
2000\end{array}$ & $\begin{array}{c}\text { RANK } \\
2011\end{array}$ & $\begin{array}{c}\text { POSICION } \\
\text { ASCENSO } \\
\text { /DESCENSO }\end{array}$ \\
\hline Piña & Alajuela & San Carlos & Pital & 0,47 & 0,51 & 352 & 393 & -41 \\
\hline Piña & Limón & Siquirres & Pacuarito & 0,44 & 0,50 & 388 & 400 & -12 \\
\hline Café & San José & Pérez Zeledón & San Pedro & 0,47 & 0,50 & 355 & 402 & -47 \\
\hline Piña/banano & Limón & Guácimo & Río Jiménez & 0,44 & 0,50 & 395 & 403 & -8 \\
\hline Piña/banano & Limón & Pococí & Roxana & 0,46 & 0,49 & 365 & 408 & -43 \\
\hline Piña & Puntarenas & Buenos Aires & Volcán & 0,45 & 0,49 & 383 & 409 & -26 \\
\hline Piña/banano & Limón & Pococí & Rita & 0,40 & 0,49 & 423 & 410 & 13 \\
\hline Banano & Limón & Matina & Matina & 0,45 & 0,49 & 384 & 413 & -29 \\
\hline Café & San José & Pérez Zeledón & Río Nuevo & 0,41 & 0,49 & 415 & 415 & 0 \\
\hline Banano & Limón & Matina & Carrandí & 0,50 & 0,48 & 310 & 417 & -107 \\
\hline Café & San José & Pérez Zeledón & Pejibaye & 0,44 & 0,47 & 389 & 421 & -32 \\
\hline Banano & Limón & Pococí & Colorado & 0,39 & 0,39 & 432 & 443 & -11 \\
\hline Banano & Limón & Limón & Valle La Estrella & 0,39 & 0,34 & 435 & 453 & -18 \\
\hline
\end{tabular}

Fuente: Elaboración propia con datos del INEC, Censos $\underline{2000}$ y $\underline{2011}$ 